\title{
Time Ordering Effects on Hydrogen Zeeman-Stark Line Profiles in Low-Density Magnetized Plasmas
}

\author{
J. Rosato, ${ }^{1}$ D. Boland, ${ }^{2}$ M. Difallah, ${ }^{2}$ Y. Marandet, ${ }^{2}$ and R. Stamm ${ }^{2}$ \\ ${ }^{1}$ Institut für Energieforschung (IEF) - Plasmaphysik, Forschungszentrum Jülich GmbH, Euratom Association, \\ Trilateral Euregio Cluster, 52425 Jülich, Germany \\ ${ }^{2}$ Physique des Interactions Ioniques et Moléculaires (PIIM), UMR 6633 Université de Provence - CNRS, Centre de St. Jérôme, \\ Case 232, 13397 Marseille Cedex 20, France \\ Correspondence should be addressed to J. Rosato, j.rosato@fz-juelich.de \\ Received 15 April 2009; Accepted 3 June 2009 \\ Academic Editor: Manuel Á. González
}

Copyright (๑) 2010 J. Rosato et al. This is an open access article distributed under the Creative Commons Attribution License, which permits unrestricted use, distribution, and reproduction in any medium, provided the original work is properly cited.

Stark broadening of hydrogen lines is investigated in low-density magnetized plasmas, at typical conditions of magnetic fusion experiments. The role of time ordering is assessed numerically, by using a simulation code accounting for the evolution of the microscopic electric field generated by the charged particles moving at the vicinity of the atom. The Zeeman effect due to the magnetic field is also retained. Lyman lines with a low principal quantum number $n$ are first investigated, for an application to opacity calculations; next Balmer lines with successively low and high principal quantum numbers are considered for diagnostic purposes. It is shown that neglecting time ordering results in a dramatic underestimation of the Stark effect on the low- $n$ lines. Another conclusion is that time ordering becomes negligible only when ion dynamics effects vanish, as shown in the case of high- $n$ lines.

\section{Introduction}

In magnetic fusion, detailed line shapes are of interest for accurate diagnostics or radiative transfer simulations. For plasma conditions and magnetic fields encountered in the divertor of present and future tokamaks, an accurate model for the line shape of the hydrogen isotopes should include Zeeman and Stark effects, and retain the dynamics of the ion-emitter interaction. Since we then have to solve a quantum time-dependent problem, understanding the role of time ordering becomes an important issue both from the fundamental and computational points of view (note, this problem is also investigated in other contexts, e.g., $[1-3]$ ). Time ordering has already been studied in the Stark broadening literature, but generally for the electron broadening [4-7]. Our aim here is to investigate the role of time ordering for the ion perturbation on hydrogen lines for plasmas with temperature in the $\mathrm{eV}$ range, and densities of about $10^{15} \mathrm{~cm}^{-3}$, conditions which are expected in the divertor of the future ITER tokamak. We recall in Section 2 the basic formalism used for line shape calculations in the presence of Stark and Zeeman effects, and briefly introduce the issue of time ordering. Line shapes in the atom's frame of reference are considered, that is, in the Doppler free case. We present in Section 3 an ab initio simulation technique able to provide accurate line shapes including all the effects of time ordering. Calculations of hydrogen line shapes of Lyman and Balmer series are presented in Section 4, with and without the effect of time ordering, and compared to calculations performed in the static ion limit. The role of time ordering and the issue of retaining it in a line shape calculation are discussed in the conclusion for lines with low and high principal quantum number of the upper state.

\section{Formalism}

According to classical textbooks or review articles (e.g., [8$10])$, a line shape in the atom's rest frame at a frequency $\omega, I(\omega)$, is given by the Fourier transform of the dipole autocorrelation function $C(t)$ : 


$$
\begin{gathered}
I(\omega)=\frac{1}{\pi} \operatorname{Re} \int_{0}^{\infty} d t C(t) e^{i \omega t}, \\
C(t)=\left\{\operatorname{Tr}\left(\rho_{n} \vec{d}_{\perp} \cdot U_{n^{\prime}}^{+}(t) \vec{d}_{\perp} U_{n}(t)\right)\right\} .
\end{gathered}
$$

Here, $n$ and $n^{\prime}$ denote the principal quantum number with respect to the initial and final states, respectively; $\vec{d}_{\perp}$ is the dipole operator projected into the polarization plane; $U_{n}(t)$ (resp., $\left.U_{n^{\prime}}(t)\right)$ is the evolution operator projected into the subspace related to the initial (resp., final) states; $\rho_{n}$ is the density operator related to the initial states; the brackets $\{\cdots\}$ stand for a statistical average over the perturbers' states and the trace $\operatorname{Tr}(\cdots)$ is performed over the atomic states. The evolution operator obeys the time-dependent Schrödinger equation

$$
i \hbar \frac{d U_{n}}{d t}(t)=\left(H_{0}-\vec{d}_{n} \cdot \vec{E}(t)\right) U_{n}(t)
$$

where $\vec{d}_{n}$ is the dipole projected into the subspace related to $n ; H_{0}$ is the atomic Hamiltonian accounting for the Zeeman effect, that is, $H_{0}=H_{\mathrm{at}}-\vec{\mu} \cdot \vec{B}$ with $\vec{\mu}, \vec{B}$ being, respectively, the magnetic moment and the magnetic field; and $\vec{E}(t)=$ $\vec{E}_{e}(t)+\vec{E}_{i}(t)$ is the time-dependent electric field created at the atom's location by both electrons $(e)$ and ions $(i)$. In our conditions of interest the electron contribution can be safely described by an impact collision operator $\Phi_{n}$. We will therefore formally replace $-\vec{d}_{n} \cdot \vec{E}_{e}(t)$ by $-i \hbar \Phi_{n}$. We will also use the interaction representation, that is, consider the evolution operator $\tilde{U}_{n}(t)=\exp \left(\left(i H_{0} / \hbar+\Phi_{n}\right) t\right) U_{n}(t)$ which obeys the following equation

$$
i \hbar \frac{d \tilde{U}_{n}}{d t}(t)=-\stackrel{\vec{d}}{n}_{n}(t) \cdot \vec{E}_{i}(t) \tilde{U}_{n}(t)
$$

where $\stackrel{\overrightarrow{\vec{d}}}{n}_{n}(t)=\exp \left(\left(i H_{0} / \hbar+\Phi_{n}\right) t\right) \vec{d}_{n} \exp \left(\left(-i H_{0} / \hbar-\Phi_{n}\right) t\right)$ is the dipole operator in the interaction representation.

The Schrödinger equation in the interaction representation (3), associated with the initial condition $\tilde{U}_{n}(0)=$ $U_{n}(0)=1$, admits as a formal solution the so-called Dyson series expansion

$$
\begin{aligned}
\tilde{U}_{n}(t)= & 1+\sum_{p \geq 1} \frac{1}{(i \hbar)^{p}} \int_{0 \leq \tau_{1} \leq \cdots \tau_{p} \leq t} \cdots \\
& \times \int d \tau_{1} \cdots d \tau_{p} \tilde{V}_{n}\left(\tau_{p}\right) \cdots \tilde{V}_{n}\left(\tau_{1}\right),
\end{aligned}
$$

where $\tilde{V}_{n}(\tau)=-\stackrel{\overrightarrow{\vec{d}}}{n}_{n}(\tau) \cdot \vec{E}(\tau)$. Following Baranger $[11,12]$, we write this solution a time-ordered exponential of a matrix

$$
\tilde{U}_{n}(t)=T \exp \left(\frac{1}{i \hbar} \int_{0}^{t} d \tau \widetilde{V}_{n}(\tau)\right) .
$$

Here, $T$ is the time ordering operator, defined by its action on a product of time-dependent operators $A\left(t_{1}\right), \ldots, A\left(t_{p}\right)$ as follows:

$T\left[A\left(t_{p}\right) \cdots A\left(t_{1}\right)\right]= \begin{cases}A\left(t_{p}\right) \cdots A\left(t_{1}\right), & \text { if } t_{p} \geq \cdots \geq t_{1}, \\ 0, & \text { otherwise. }\end{cases}$
Because of the presence of the $T$-operator in (5), the evolution operator cannot in general be described by a pure exponential of a matrix since the $\tilde{V}_{n}$-operator does not commute with itself at different times. The time ordering operator can only be dropped out in the limiting case where the electric field does not evolve significantly during the time of interest (quasistatic limit). In the following we will address the general case by using a simulation code.

\section{Assessment of Time Ordering by Computer Simulations}

3.1. The Numerical Simulation Method. The purpose of ab initio simulations is to numerically reproduce the motion of the charged particles in the plasma so as to obtain the time-dependent electric microfield $\vec{E}_{i}(t)$. Essentially, a numerical simulation consists of (i) the calculation of a set of realizations for the electric field; (ii) the numerical integration of the Schrödinger equation for each realization; (iii) the average of the evolution operators on a set of realizations and the Fourier transform of the autocorrelation function. In the simulations performed for this work, we use a code [13] developed according to the method reported in [14]. We consider that the ions move along straight line trajectories with constant velocities, sampled among the particles according to an equilibrium Maxwell distribution function. The electrons (whose contribution to line broadening is mostly negligible for our conditions of interest) are not simulated here, but are described with an impact collision operator (see previous section). The treatment of the correlations between ions and electrons is retained by using Debye screened fields. The largeness of the ratio $\rho_{\mathrm{Li}} / \lambda_{\mathrm{D}}$ between the ion Larmor radius and the Debye length (of about 40 at $N=10^{14} \mathrm{~cm}^{-3}, T=$ $1 \mathrm{eV}, B=5 \mathrm{~T}$, i.e., typical conditions expected in the ITER divertor) ensures the assumption of straight line trajectories. A cubic cell with periodic boundary conditions is considered. For each history of the electric field, the code solves the time-dependent Schrödinger equation for the evolution operator $\tilde{U}_{n}(t)$ according to the algorithm $\tilde{U}_{n}(t+\Delta t)=$ $\tilde{U}_{n}(t+\Delta t, t) \widetilde{U}_{n}(t)$, with $\widetilde{U}_{n}(t+\Delta t, t)$ being the infinitesimal evolution operator between times $t$ and $t+\Delta t$. The latter operator is not affected by time ordering if the time step $\Delta \mathrm{t}$ is sufficiently small, and hence can be evaluated by a matrix exponential

$$
\tilde{U}_{n}(t+\Delta t, t)=\exp \left(\frac{1}{i \hbar} \Delta t \tilde{V}_{n}(t)\right)
$$

In the code, we use this property and calculate the exponential according to the scaling squaring method (e.g., [15]). This method is also used for the calculation of the expression of $\tilde{U}_{n}(t)$ not accounting for time ordering, given by the exponential of $\int_{0}^{t} d t^{\prime} \tilde{V}_{n}\left(t^{\prime}\right) / i \hbar$.

3.2. Line Shape Calculations. Time ordering should play a role on lines which are affected by ion dynamics, that is, with a low upper principal quantum number $n$. To illustrate 


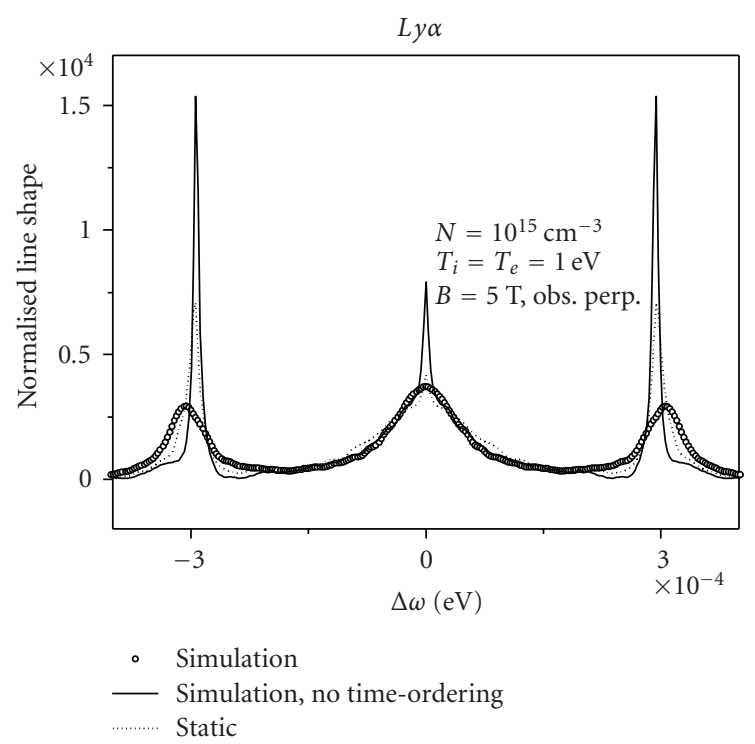

(a)

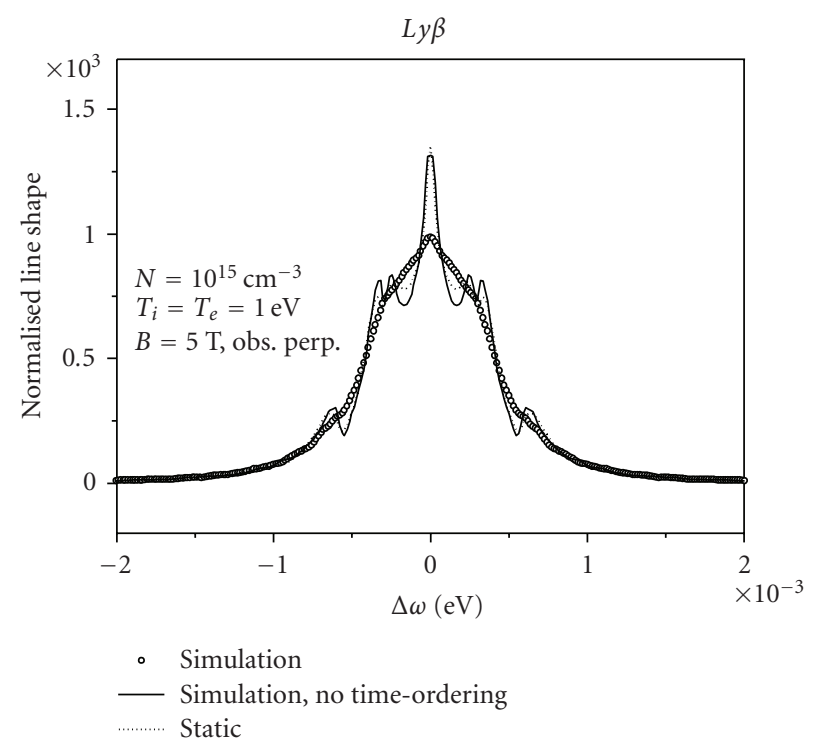

(b)

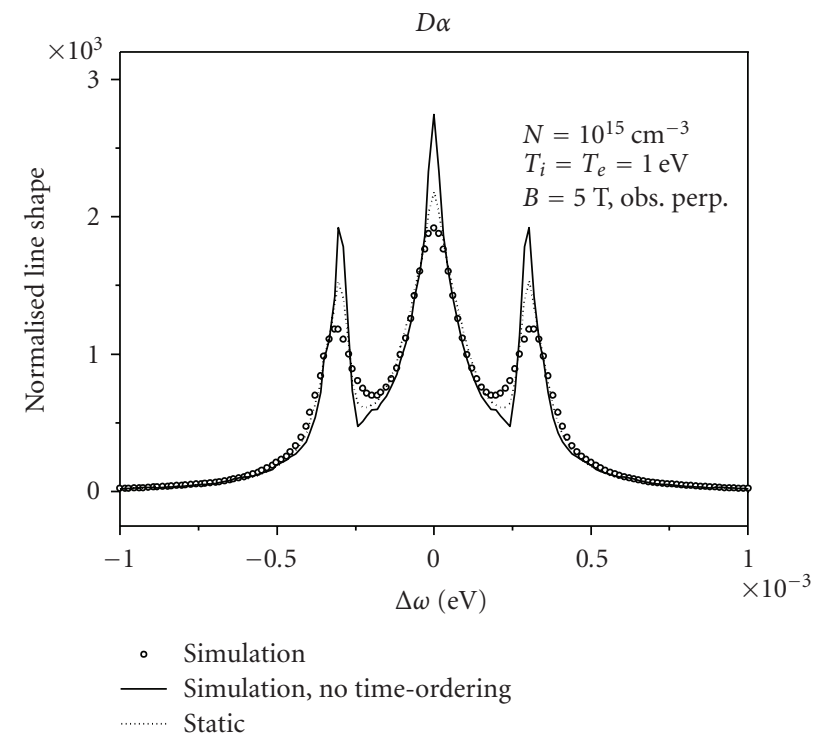

(c)

Figure 1: Zeeman-Stark profiles of (a) $L y \alpha$, (b) $L y \beta$, and (c) $D \alpha$, obtained at typical divertor conditions with the simulation method accounting for time ordering (circles) or not (full line), and with the quasistatic approximation (dots). In each case, neglecting time ordering leads to a dramatic underestimation of the line broadening, and provides more structure.

this point, we have successively calculated $L y \alpha, L y \beta$ (whose shapes are involved in the calculations of divertor plasma opacity), and $D \alpha$ (which is commonly observed in tokamak experiments and routinely used for diagnostic purposes). Figures 1(a), 1(b), and 1(c), show, for each line profile, the result obtained with simulations retaining time ordering or not, at $N=10^{15} \mathrm{~cm}^{-3}, T_{e}=T_{i}=1 \mathrm{eV}, B=5 \mathrm{~T}$, and in perpendicular observation. The profiles resulting from the quasistatic approximation are also plotted. In each case, the Zeeman effect is important and leads either to a Lorentz triplet structure $(L y \alpha, D \alpha)$ or to an additional broadening $(L y \beta)$. As can be seen, neglecting time ordering leads to a dramatic underestimation of the Stark effect. The Zeeman components of $L y \alpha$ and $D \alpha$ are much narrower than those obtained both with the exact solution and the quasistatic approximation (note, by a factor of $\sim 3-4$ in the case of the lateral components of $L y \alpha$ ), and the Zeeman-Stark pattern becomes visible on $L y \beta$ when time ordering is neglected. Another gain of structure is provided by the apparition of Stark components, in particular on $L y \beta$ where a splitting of the lateral Zeeman components due to Stark effect is clearly visible.

The underestimation of the Stark effect can be explained by noting that, in the solution of Schrödinger's equation neglecting time ordering, the matrix exponential essentially involves the time average of the electric field $(1 / t) \int_{0}^{t} d t^{\prime} \vec{E}_{i}\left(t^{\prime}\right)$. 


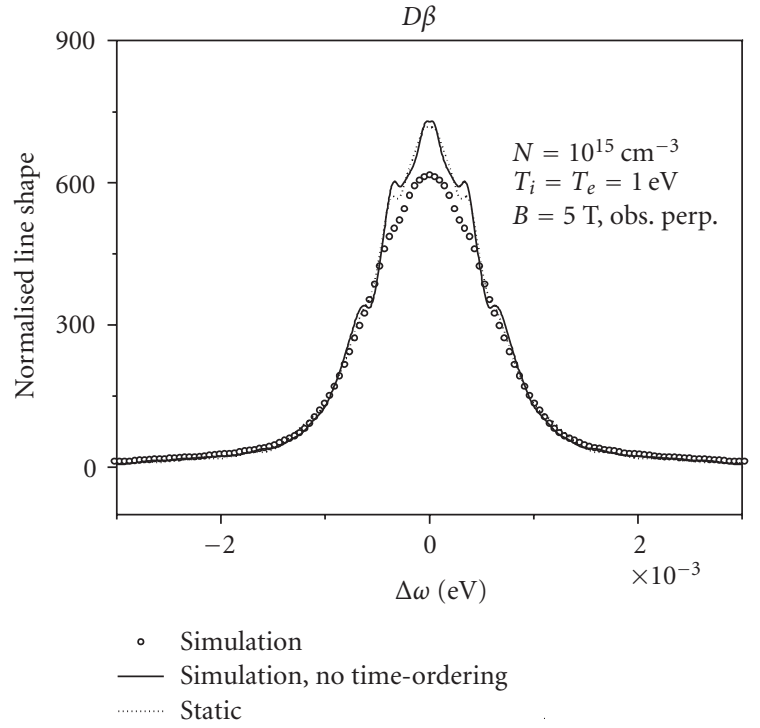

(a)

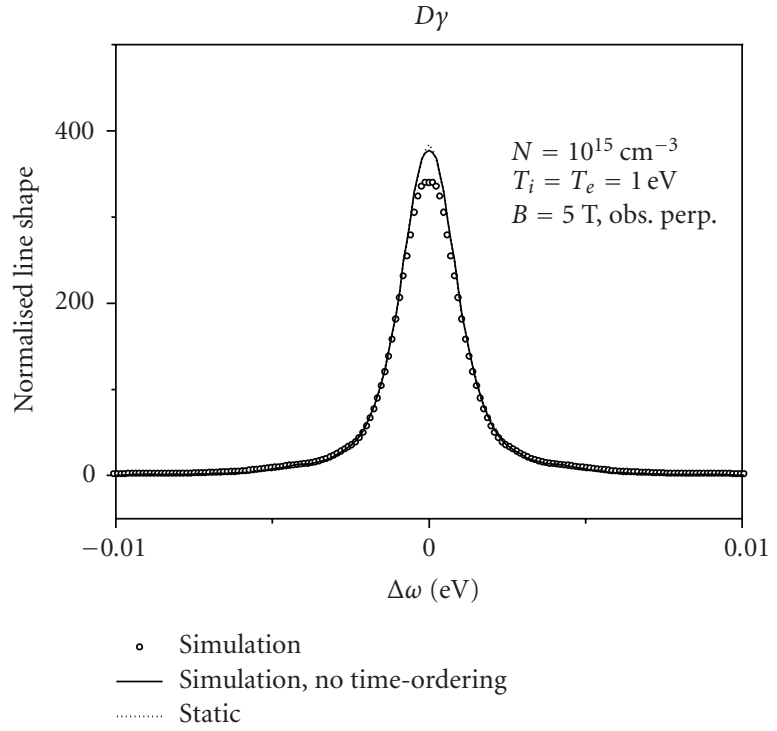

(b)

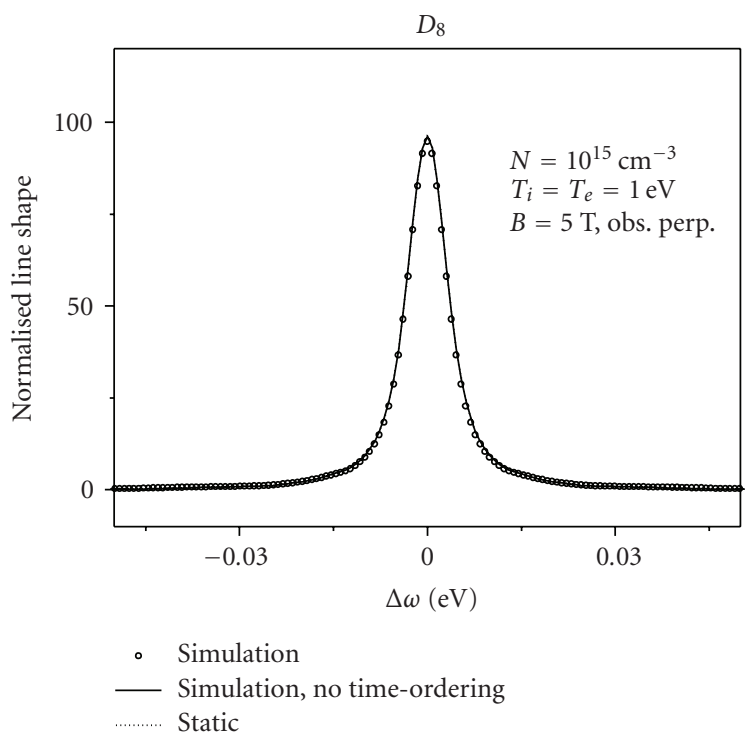

(c)

Figure 2: Profiles of (a) $D \beta$, (b) $D \gamma$, and (c) $D_{8}$. The effect of time ordering decreases as the upper principal quantum number increases, that is, as the quasistatic limit for ions is approached.

This quantity vanishes on a time scale smaller than the time of interest, a quantity usually defined as the inverse of the line width. Therefore, except for very large frequencies (which correspond to short times), the emitter-ion interaction potential is very small and the resulting line shape becomes very narrow. The line shape is even narrower than that obtained using the quasistatic approximation because the cancellation of the average electric field, equivalent to a cancellation of the evolution operator oscillations, leads to a slower decorrelation of the atomic dipole. The deviation should be smaller for lines with a higher upper principal quantum number $n$, because ion dynamics is less important in this case. This is indeed illustrated in Figure 2, where a plot of (a) $D \beta$, (b) $D \gamma$, and (c) $D_{8}$ are presented. For these lines, the ratio $t_{i} / t_{c}$ between the time of interest at half maximum $t_{i}$ and the collision time $t_{c}$ is $14 \%, 9 \%$, and $0.2 \%$, respectively. As shown, the role of time ordering becomes less and less important as this ratio decreases, that is, as the ions become static.

\section{Conclusion}

We have addressed the role of time ordering on hydrogen Zeeman-Stark profiles in low-density plasmas, for typical conditions of tokamak divertors. With numerical simulations, we have shown that neglecting time ordering on lines with a low upper principal quantum number leads to 
strong deviations, with a systematic underestimate of the Stark width of the Zeeman components. This is interpreted in terms of the time averaged electric field, namely, the latter rapidly vanishes during the decorrelation of the atomic dipole so that the resulting effective Stark effect is reduced. Conversely, we have shown that the deviations are weak on lines with a higher upper quantum number, merely because they are much less affected by ion dynamics. This result, of interest for spectroscopy of magnetic fusion experiments, shows that: (i) the development of line shape models including ion dynamics for Monte Carlo investigations of radiative transfer (e.g., [16]) requires to account for time ordering; (ii) in a similar way, time ordering must be accounted for in the Stark broadening models used for $D \alpha$ passive spectroscopy diagnostics; (iii) obviously, the effect of time ordering disappears as one approaches the validity conditions of the static ion approximation, and this is clearly the case for high$n$ lines in our divertor conditions. A possible extension of our work would consist of a parameterization of the role of time ordering on low- $n$ lines, for diagnostic as well as for radiative transfer calculation purposes.

\section{Acknowledgments}

This work is supported by an EFDA Fellowship contract, by the French Federation on Magnetic Fusion Research (project "Radiation Absorption Effects"), by the French Research National Agency (Project "PHOTONITER", Contract ANR07-BLAN-0187-01), and by the collaboration PIIM/CEA Cadarache (Contract LRC DSM 99-14).

\section{References}

[1] J. H. McGuire, A. L. Godunov, K. K. Shakov, et al., "Time ordering in multi-electron dynamics," Journal of Physics B, vol. 36, no. 2, pp. 209-216, 2003.

[2] L. Kaplan, K. K. Shakov, A. Chalastaras, M. Maggio, A. L. Burin, and J. H. McGuire, "Time ordering in kicked qubits," Physical Review A, vol. 70, no. 6, Article ID 063401, 2004.

[3] D. Lauvergnat, S. Blasco, X. Chapuisat, and A. Nauts, "A simple and efficient evolution operator for time-dependent Hamiltonians: the Taylor expansion," Journal of Chemical Physics, vol. 126, no. 20, Article ID 204103, 2007.

[4] C. R. Vidal, J. Cooper, and E. W. Smith, "Hydrogen Stark broadening calculations with the unified classical path theory," Journal of Quantitative Spectroscopy and Radiative Transfer, vol. 10, no. 9, pp. 1011-1063, 1970.

[5] J. T. Godfrey, C. R. Vidal, E. W. Smith, and J. Cooper, "Effect of time ordering in the unified theory," Physical Review A, vol. 3, no. 5, pp. 1543-1546, 1971.

[6] L. J. Roszman, "Effects of time ordering on plasma-broadened hydrogen profiles," Physical Review Letters, vol. 34, no. 13, pp. 785-788, 1975.

[7] M. A. Gunderson, G. C. Junkel-Vives, and C. F. Hooper Jr., "A comparison of a second-order quantum mechanical and an all-order semi-classical electron broadening model," Journal of Quantitative Spectroscopy and Radiative Transfer, vol. 71, no. 2-6, pp. 373-382, 2001.

[8] V. S. Lisitsa, "Stark broadening of hydrogen lines in plasmas," Soviet Physics - Uspekhi, vol. 20, p. 603, 1977.
[9] H. R. Griem, Principles of Plasma Spectroscopy, Cambridge University Press, New York, NY, USA, 1997.

[10] E. Oks, Stark Broadening of Hydrogen and Hydrogenlike Spectral Lines in Plasmas, Alpha Science, Oxford, UK, 2006.

[11] M. Baranger, "Problem of overlapping lines in the theory of pressure broadening," Physical Review, vol. 111, no. 2, pp. 494504, 1958.

[12] M. Baranger, "General impact theory of pressure broadening," Physical Review, vol. 112, no. 3, pp. 855-865, 1958.

[13] J. Rosato, Y. Marandet, H. Capes, et al., "Stark broadening of hydrogen lines in low-density magnetized plasmas," Physical Review E, vol. 79, no. 4, Article ID 046408, 2009.

[14] R. Stamm, E. W. Smith, and B. Talin, "Study of hydrogen Stark profiles by means of computer simulation," Physical Review A, vol. 30, no. 4, pp. 2039-2046, 1984.

[15] C. Moler and C. Van Loan, "Nineteen dubious ways to compute the exponential of a matrix, twenty-five years later," SIAM Review, vol. 45, no. 1, pp. 3-49, 2003.

[16] D. Reiter, V. Kotov, P. Börner, K. Sawada, R. K. Janev, and B. Küppers, "Detailed atomic, molecular and radiation kinetics in current 2D and 3D edge plasma fluid codes," Journal of Nuclear Materials, vol. 363-365, no. 1-3, pp. 649-657, 2007. 


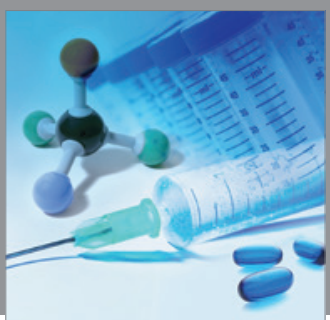

International Journal of

Medicinal Chemistry

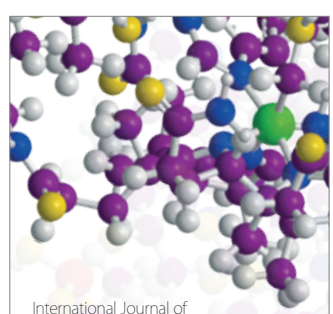

Carbohydrate Chemistry

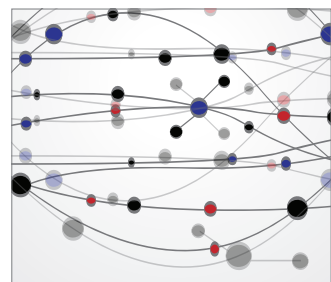

The Scientific World Journal
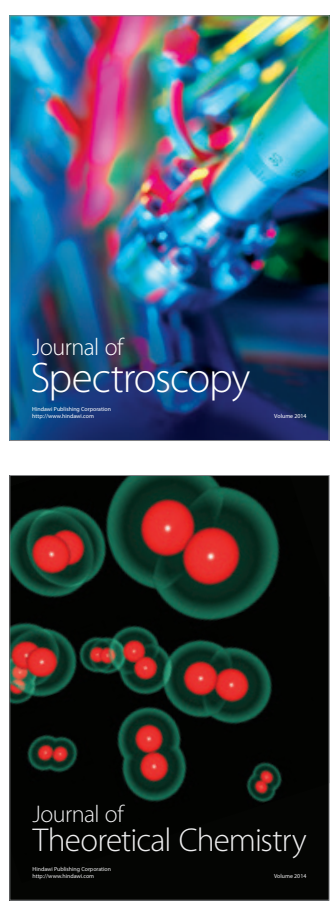
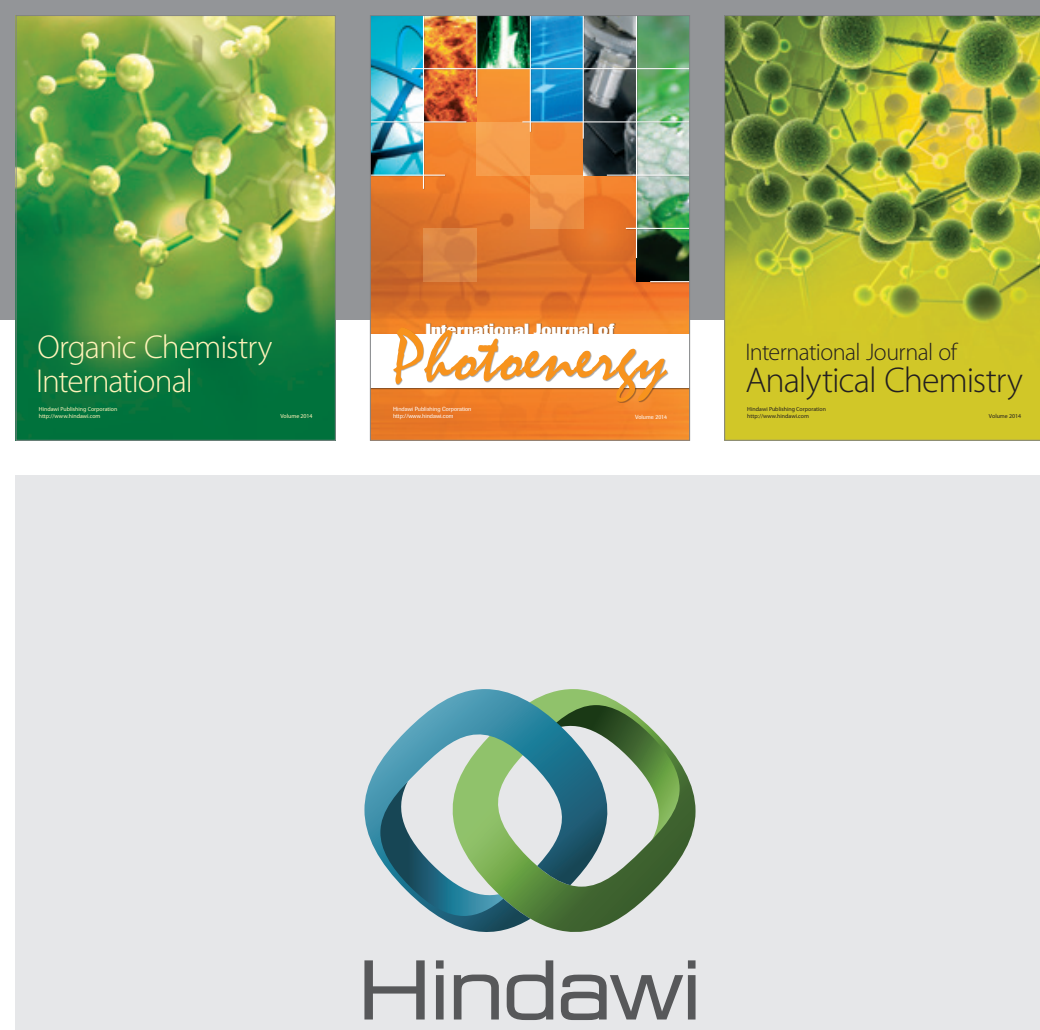

Submit your manuscripts at

http://www.hindawi.com
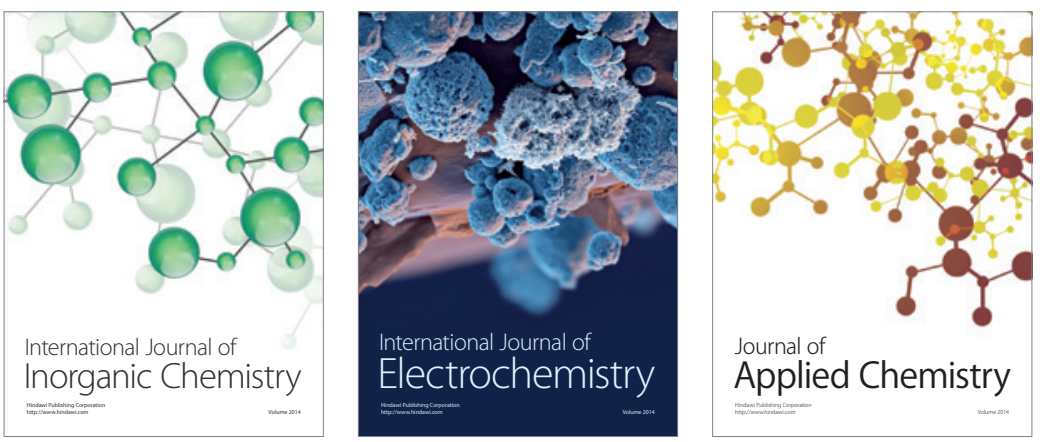

Journal of

Applied Chemistry
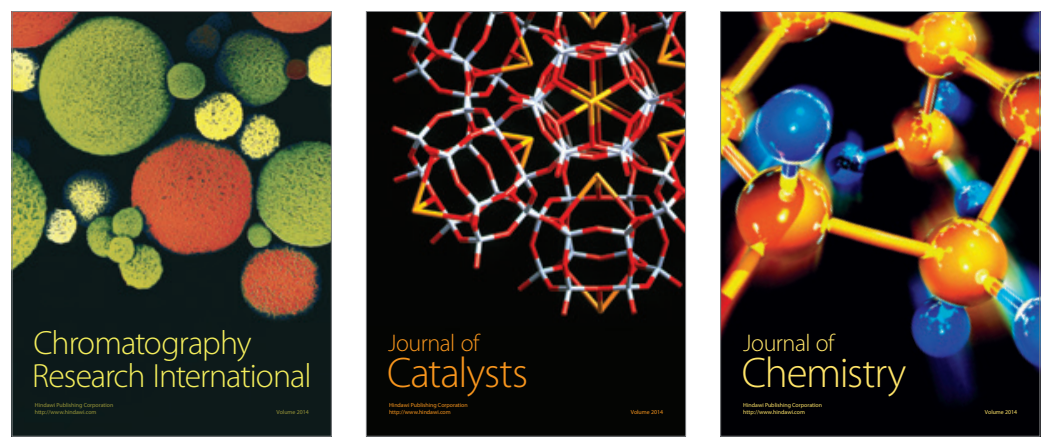
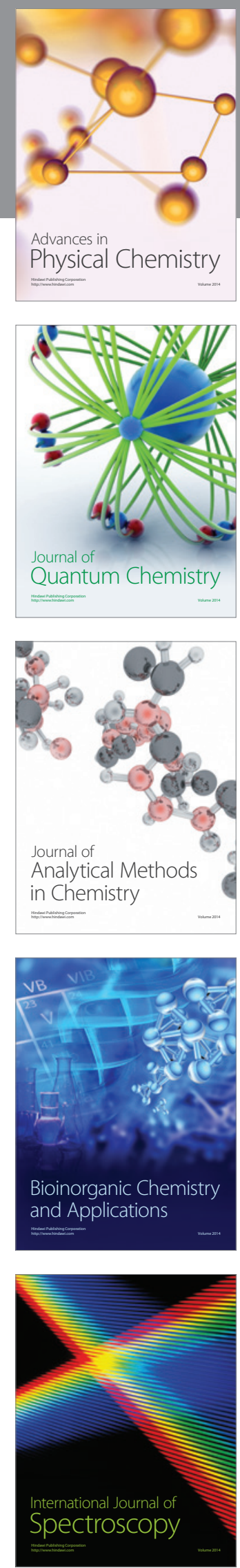